\title{
Educational Choice, Endogenous Inequality and Economic
}

\section{Development}

\author{
Rainer Andergassen ${ }^{1}$, Franco NARdini $^{2 *}$ \\ ${ }^{1}$ Department of Economics, University of Bologna \\ P.zza Scaravilli 2, 40126 Bologna (Italy) \\ ${ }^{2}$ Department of Mathematics for the Social Sciences, University of Bologna \\ Viale Filopanti 5, 40126 Bologna (Italy)
}

March 2004

Abstract. This paper investigates the mechanics through which wealth may, in the long run, trickle down from the rich to the poor. In the presence of indivisibilities in investment of human capital and impossibility of borrowing money, investment in education is financed through an intergenerational transfer. In an OLG model where aggregate production requires capital and both skilled and unskilled labour, it is shown that the long run equilibrium outcome depends on the values of few key parameters. A complete characterisation of the steady state is provided. Under some configurations of the parameter values a unique invariant equilibrium exists where inequality vanishes asymptotically. Under others, multiple equilibria exist and the equilibrium outcome crucially depends on the initial conditions of the system. These equilibria are characterised by an negative relationship between inequality and economic development.

KEYwORDS: inequality, trickle down, economic development

JEL: O11, O15

\footnotetext{
*Corresponding author; Tel: +39 0512094364, Fax +39 0512094367. E-mail addresses: anderga@economia.unibo.it (R. Andergassen), nardini@dm.unibo.it (F. Nardini).
} 


\section{Introduction}

In presence of imperfect credit markets, intergenerational transfers within the family are particularly important for economic development and wealth inequality. Investment in education, which is largely indivisible, has to be financed out of the savings of parents and consequently the income distribution crucially matters both at the individual and at the aggregate level. At the individual level an unskilled dynasty may be unable to escape its inferior state leading to persistent inequality. At the aggregate level, underinvestment in education may result in underdevelopment and poor economic performance. This is all the more relevant today as technological capability depends on the availability of skilled workers.

We build an overlapping generation general equilibrium model where each generation lives only for two periods. When young, individuals face the opportunity to invest in education. After this, they supply inelastically one unit of labour. Individuals when young are credit constrained. In particular, we assume that they are unable to borrow money. Thus, investment in education can be financed only thanks to intergenerational transfers, which are due to "warm glow" preferences of parents over bequests (see Andreoni, 1989 and Aghion and Bolton, 1997). Investment in education is indivisible and is assumed to be a fixed proportion of aggregate output. On the other hand, we assume that individuals are able to lend money at the endogenous equilibrium interest rate which depends on the marginal productivity of capital. Finally, when old they consume and determine the size of the intergenerational transfer.

We assume that aggregate production requires capital and both skilled and unskilled labour. Skilled and unskilled labour can be either gross complements or gross substitutes (as in Acemoglu, 2002). We derive long run equilibria characterising steady states. We focus on the mechanics through which wealth may, in the long run, trickle down from the rich (skilled) to the poor (unskilled) and its consequences on aggregate wealth.

We identify four key parameters: the size of the intergenerational transfer, the relative impor- 
tance of skilled labour in the production function, the elasticity of substitution between skilled and unskilled labour and the relative cost of education. We find a threshold level for the relative cost of education above which trickle down is always unfeasible irrespectively of the initial conditions of the economy. On the other hand, below this threshold level the possibility of a trickle down depends on the value of the other key parameters as well as on the initial conditions of the system. In particular, we find that the larger is the size of the intergenerational transfer or the lower is the relative importance of the skilled labour in the production function, the larger is the set of initial conditions such that trickle down eventually occurs. Thus, initial conditions crucially matter, and an economy may well be trapped into an equilibrium with persistent inequality and underdevelopment. We show that, under certain conditions, in the case of multiple equilibria, these can be ordered in terms of aggregate output. In this case, an negative relationship between inequality and aggregate wealth can be established. In other words, the greater the inequality, the more underdeveloped the country is.

The system undergoes a structural change as skilled and unskilled labour switches from gross complements to gross substitutes. In particular, we show that if factors are gross substitutes, then there exists always values for the relative cost of education which allows trickle down irrespectively of the initial conditions. Thus, for sufficiently low values of the relative cost, a unique, invariant equilibrium exists where inequality vanishes asymptotically. For larger values of the relative costs, besides the trickle down equilibrium, a continuum of equilibria appears, where inequality persists forever. On the other hand, if factors are gross complements, then for each level of the relative cost of education, trickle down equilibrium always coexists with a continuum of equilibria characterised by an negative relationship between (persistent) inequality and economic developement.

The present paper contributes to the literature which relates the dynamics of the income distribution to the macroeconomic performance of an economy. Galor and Zeira (1993) present a model with capital market imperfections and indivisibilities in human capital investments. The interplay between these two features polarises the economy into two different classes: a rich one 
and a poor one. In a related paper Banerjee and Newman (1993) investigate the link between income distribution and occupational choice in a model with borrowing constraints. The authors show that the peace of economic development and the degree of inequality crucially depends on the initial conditions of the economy ${ }^{1}$.

Our paper is closely related to Mookherjee and Ray (2003) where the authors investigate the conditions for sustained and persistent inequality. One key difference between our and their paper concerns intergenerational transfers. In Mookherjee and Ray (2003) individuals have dynastic preferences (as in Barro (1974)). Individuals derive utility from consumption and the utility of their offspring. As a consequence, professions in their paper are bequeath to their offspring. They show that equal steady states cannot exist and thus in every steady state consumption and utility is different across households in every generation ${ }^{2}$.

The remaining part of the paper is organised as follows. In Section 2 we briefly outline the main features of the model. In Section 3 we derive the dynamics of the endowments of the skilled and unskilled cohorts. In Section 4 we derive the steady state of the model, where we focus in particular on the skill premium condition and on the education investment feasibility constraints. Further, the possibility of trickle down and its consequences in terms of aggregate production are investigated. Finally, Section 5 concludes the paper.

\section{The model}

We consider an overlapping generation model (OLG). The population of each generation is a unit mass.

Output can either be used for consumption, investment in physical capital or for the production of education. We first outline the microeconomic setup (Section 2.1) where the problem of the

\footnotetext{
1 In Galor and Zeira (1993) and Banerjee and Newman (1993) the interest rate is fixed and exogenously given. Piketty (1997), Aghion and Bolton (1997) and Matsuyama (2002) study the relationship between endogenous wealth distribution and inequality in the case of credit market imperfections where the interest rate adjusts endogenously to maintain the balance between the demand and supply of credit.

${ }^{2}$ In Mookherjee and Ray (2002) this result is somehow relaxed due to the possibility of financial bequests.
} 
representative agent is stated. In Section 2.2 the macroeconomic framework is described. In particular, the aggregate production function and the capital accumulation constraint are described.

\subsection{Microeconomic setup}

Each generation has utility function

$$
U\left(c_{t}^{i}, z_{t+1}^{i}, \mu_{t}^{i}, E_{t+1}^{i}\right)=\beta \log c_{t}^{i}+\log z_{t+1}^{i}+\alpha \log E_{t+1}^{i}
$$

where $c$ indicates consumption when young, $z$ indicates consumption when old and $E$ indicates the intergenerational transfer.

Each individual has to decide whether to invest in education or not. Investment in education takes place when young. The cost of education for each individual at time $t$ is $F_{t}$. Individuals when young are credit constrained and are not able to borrow. On the other hand, we assume that individuals are able to lend money at the equilibrium interest rate which depends on the marginal productivity of capital. Thus, education costs have to be covered out of initial endowments $E_{t}^{i}$, which is an intergenerational transfer (or bequest). If $\mu_{t}^{i}=1$ the individual invests in education, while $\mu_{t}^{i}=0$ he does not. $w^{H}$ and $w^{L}$ are the wages of the skilled and unskilled workers, respectively. Thus, the budget constraints are as follows

$$
\begin{gathered}
c_{t}^{i}=\mu_{t}^{i} w_{t}^{H}+\left(1-\mu_{t}^{i}\right) w_{t}^{L}+E_{t}^{i}-\mu_{t}^{i} F_{t}-s_{t}^{i} \\
z_{t+1}^{i}=R_{t+1} s_{t}^{i}-E_{t+1}^{i}
\end{gathered}
$$

where $s_{t}^{i}$ indicates savings, $R_{t}$ is the interest rate.

Investment in education is feasible only if

$$
E_{t}^{i} \geq F_{t}
$$

which is the education investment feasibility constraint.

We assume that, in order to educate one individual, a fraction $f$ of total output has to be used 
as input. More formally, we assume that

$$
F_{t}=f Y_{t}
$$

\subsection{Macroeconomic setup}

$H_{t}$ and $L_{t}$ indicates skilled and unskilled workers, respectively, where $H_{t}+L_{t}=1$.

Aggregate production function is

$$
Y_{t}=K_{t}^{\gamma} N_{t}^{\frac{1-\gamma}{\rho}}
$$

where $N_{t}=\eta H_{t}^{\rho}+(1-\eta) L_{t}^{\rho}$. We assume that, $\eta \in\left(\frac{1}{2}, 1\right)$, i.e. skilled workers are relatively more important for aggregate production, than unskilled ones, and $\rho \leq 1$. For $\rho=1$ skilled and unskilled workers are perfect substitutes, for $\rho=-\infty$ the two are perfect complements. Following Acemoglu (2002), for $0 \leq \rho<1$ we refer to the two factors as gross substitutes, while for $\rho<0$ we refer to them as gross complements.

Equilibrium wages and equilibrium interest rates are given by

$$
\begin{gathered}
w_{t}^{H}=(1-\gamma) \eta \frac{Y_{t}}{N_{t}} H_{t}^{\rho-1} \\
w_{t}^{L}=(1-\gamma)(1-\eta) \frac{Y_{t}}{N_{t}} L_{t}^{\rho-1} \\
R_{t}=\gamma \frac{Y_{t}}{K_{t}}
\end{gathered}
$$

Capital accumulation is given by

$$
K_{t+1}=\int_{0}^{1} s_{t}^{i} d i
$$

\subsection{Savings, intergenerational transfers and the skill premium condition}

From the first order condition (FOC) for the choice of bequests we obtain

$$
E_{t+1}^{i}=b R_{t+1} s_{t}^{i}
$$

where $b=\frac{\alpha}{1+\alpha}$ and from (2) we obtain $z_{t+1}^{i}=(1-b) R_{t+1} s_{t}^{i}$. FOC for savings yields

$$
s_{t}^{i}=\frac{\mu_{t}^{i} w_{t}^{H}+\left(1-\mu_{t}^{i}\right) w_{t}^{L}+E_{t}^{i}-\mu_{t}^{i} F_{t}}{1+\beta}
$$


Investment in eduction is desirable only if

$$
w_{t}^{H}-w_{t}^{L} \geq F_{t}
$$

Using (6), this latter condition leads to the skill premium condition (SPC):

$$
(1-\gamma)\left[\eta H_{t}^{\rho-1}-(1-\eta) L_{t}^{\rho-1}\right] \geq f N_{t}
$$

Given condition (10) investment in education is feasible only if the inherited wealth is large enough, i.e. (3) is satisfied.

\section{Dynamics of endowments for educated and non-educated cohorts}

We study the dynamics of the endowments of each generation in a situation where skilled individuals remain skilled and unskilled remain unskilled. $E_{t}^{H}$ indicates inherited wealth of skilled individuals, i.e. $\mu_{t}^{i}=1$ for each $t$, while $E_{t}^{L}$ indicates the inherited wealth of unskilled individuals, i.e. $\mu_{t}^{i}=0$ for each $t$.

Consider first $E_{t}^{L}$. Using (8), (6) and (9) we have

$$
E_{t+1}^{L}=\frac{b}{1+\beta} \gamma \frac{Y_{t+1}}{K_{t+1}}\left((1-\gamma)(1-\eta) \frac{Y_{t}}{N_{t}} L_{t}^{\rho-1}+E_{t}^{L}\right)
$$

Equivalently, the dynamics for $E_{t}^{H}$ are

$$
E_{t+1}^{H}=\frac{b}{1+\beta} \gamma \frac{Y_{t+1}}{K_{t+1}}\left((1-\gamma) \eta \frac{Y_{t}}{N_{t}} H_{t}^{\rho-1}-f Y_{t}+E_{t}^{H}\right)
$$

The dynamics of capital accumulation (7) can be rewritten as

$$
K_{t+1}=H_{t} \frac{w_{t}^{H}+E_{t}^{H}-F_{t}}{1+\beta}+L_{t} \frac{w_{t}^{L}+E_{t}^{L}}{1+\beta}
$$

Using (6) we obtain

$$
K_{t+1}=\frac{(1-\gamma) Y_{t}+H_{t}\left(E_{t}^{H}-f Y_{t}\right)+L_{t} E_{t}^{L}}{(1+\beta)}
$$

The dynamics of the economy are described by (11), (12) and (13). 
From the endowment dynamics (8), the capital accumulation (7) and the interest rate (6) we can rewrite the aggregate production as follows

$$
Y_{t}=\frac{1}{\gamma b} \int_{0}^{1} E_{t}^{i} d i
$$

Using (14), the cost of education (4) reads

$$
F_{t}=\frac{f}{\gamma b} \bar{E}_{t}
$$

where $\bar{E}_{t}$ is the average wealth at time $t$ inherited by individuals, defined as $\bar{E}_{t}=\int_{0}^{1} E_{t}^{i} d i$. Further, using (8), (9), (6) and (14) individual inherited wealth dynamics is given by

$$
\xi_{t}=\frac{\left(\eta(1-\gamma) H^{\rho-1}-f N_{t-1}\right) \bar{E}_{t-1}+\gamma b N_{t-1} E_{t-1}^{H}}{(1-\gamma)(1-\eta) L^{\rho-1} \bar{E}_{t-1}+\gamma b N_{t-1} E_{t-1}^{L}}
$$

where $\xi_{t} \equiv \frac{E_{t}^{H}}{E_{t}^{L}}$ and $p E_{t}^{H}=\int_{0}^{1} E_{t}^{i} \mu_{t}^{i} d i$ and $(1-p) E_{t}^{L}=\int_{0}^{1} E_{t}^{i}\left(1-\mu_{t}^{i}\right) d i . E_{t}^{H}$ and $E_{t}^{L}$ are the endowments of the representative skilled and unskilled individuals respectively, if all individuals initially had the same endowments.

In the next Section we study the steady state of the economy.

\section{Steady state}

In order to obtain economically meaningful results, we assume for the following that $f<1-\gamma$. Equation (16) allows for the analysis of the steady state.

Lemma 1 The difference equation

$$
\xi_{t}=\frac{\left(\eta(1-\gamma) p^{\rho-1}-N_{t-1} f\right)\left(p \xi_{t-1}+(1-p)\right)+\gamma b N_{t-1} \xi_{t-1}}{(1-\gamma)(1-\eta)(1-p)^{\rho-1}\left(p \xi_{t-1}+(1-p)\right)+\gamma b N_{t-1}}
$$

has one globally stable fixed point

$$
\widetilde{\xi}=(1-p)^{1-\rho}\left[\frac{\eta}{1-\eta} p^{\rho-1}-f \frac{\eta p^{\rho}+(1-\eta)(1-p)^{\rho}}{(1-\gamma)(1-\eta)}\right] .
$$

Proof. In the Appendix.

If for every $i$ either $\mu_{t}^{i}=1$ for all $t$ or $\mu_{t}^{i}=0$ for all $t$, then (18) is approached asymptotically and $E_{t}^{i} \underset{t \rightarrow+\infty}{\longrightarrow} E^{H}$ if $\mu_{t}^{i}=1$ for all $t$, while and $E_{t}^{i} \underset{t \rightarrow+\infty}{\longrightarrow} E^{L}$ if $\mu_{t}^{i}=0$ for all $t$. If this is not the 
case, then the trajectory will eventually hit one of the above mentioned bounds (10) and (3). On the one hand it may happen that for some $t$ also individuals of unskilled dynasties can invest in education; on the other hand individuals belonging to skilled dynasties may happen to receive less than they need to invest in education. We are going the examine these cases in detail.

\subsection{Skill premium condition and feasibility of investment in human cap- ital}

In this Section we further characterise the steady state properties of the skill premium condition and of the investment feasibility constraints. The former states the conditions such that, in the steady state, investment in education pays off from the individual point of view. The latter specifies conditions such that, in the steady state, educated cohorts can afford - cannot afford to invest in education and non-educated cohorts cannot afford - can afford to invest in education.

The following lemma characterises the skill premium condition (10).

Lemma 2 Given $H=p$ and $L=1-p$, the skill premium condition (10) is satisfied as long as

$$
f \leq \sigma(p) \equiv(1-\gamma) \frac{\eta p^{\rho-1}-(1-\eta)(1-p)^{\rho-1}}{\eta p^{\rho}+(1-\eta)(1-p)^{\rho}}
$$

where the function $\sigma(p)$ is (i) increasing in $\eta$; (ii) decreasing in $p \in(0,1)$ and (iii) increasing in $\rho$ for $p \in\left(\frac{1}{2}, 1\right)$ and decreasing in $\rho$ for $p \in\left(0, \frac{1}{2}\right)$.

Proof. In the Appendix.

Lemma 2 states that the skill premium is larger, the larger is $\eta$ and the lower is $p$. The intuition for this result is straightforward: the larger is $\eta$, the more important are the skilled workers in the production function and thus the larger is the relative compensation of skilled workers. On the other hand, the larger is $p$, the more abundant are skilled workers, and consequently the lower is their relative compensation. For $\rho \rightarrow-\infty$ skilled and unskilled workers are perfect complements and consequently the unique equilibrium is $p=\frac{1}{2}$. For $p<\frac{1}{2}$, skilled workers are relatively abundant, and consequently they are relatively cheap. An increase in the substitutability between skilled and unskilled workers increases the value of the skilled workers and consequently its skill 
premium. On the other hand, if $p<\frac{1}{2}$ skilled workers are relatively scarce, and consequently relatively costly. Thus, a reduction in the substitutability between skilled and unskilled workers increases further the relative cost of skilled workers, i.e. the skill premium.

The following lemma characterises the education investment feasibility constraints.

Lemma 3 According to the education investment feasibility constraint (15), the following conditions have to be satisfied: (a) $H=1$ is an equilibrium as long as $f<\gamma b$; (b) $H=p$ is an equilibrium as long as $\xi_{t}>\xi^{*} \equiv \frac{f(1-p)}{\gamma b-p f}$ and $\xi_{t}>\xi^{* *} \equiv \frac{\gamma b-f(1-p)}{f p}$; (c) $H=0$ is an equilibrium as long as $f>\gamma b$.

Proof. In the Appendix.

According to Lemma 3, trickle down is a feasible equilibrium only if the relative cost of education $f$ is sufficiently low, i.e. $f<\gamma b$. In particular, if $f>\gamma b$, i.e. the relative cost is too large, then trickle down equilibrium is never feasible. The condition on the feasibility of the trickle down equilibrium is not sufficient to guarantee that it will eventually emerge as an equilibrium. In the next section we further investigate the possibility of the trickle down equilibrium.

\subsection{Trickle down of wealth}

The steady state can be described using the three functions $\sigma(p), \varphi(p)$ and $\psi(p) \cdot \sigma(p)$ is the skill premium condition (19) and describes the values of $f$ as a function of $p$, such that the gain from investing in education is equal to its costs. Its properties have been described in Lemma (2). For a given the value of $p, \varphi(p)$ and $\psi(p)$ are the values of $f$ such that the endowment of educated and non-educated individuals is just enough to pay the investment in education. More formally, $\varphi(p)$ and $\psi(p)$ describe the locus of $f$ such that in the steady state $\widetilde{\xi}=\xi^{*}$ and $\widetilde{\xi}=\xi^{* *}$, respectively. The following Lemma describes the main features of $\sigma(p), \varphi(p)$ and $\psi(p)$.

Lemma 4 (i) For $f=\gamma b$, there exists a unique $p^{*} \in(0,1)$ such that $\gamma b=\sigma\left(p^{*}\right)=\psi\left(p^{*}\right)=$ $\varphi\left(p^{*}\right)$.

(ii) For each $0<p<p^{*}$ the we have that $\sigma(p)>\varphi(p)>\gamma b>\psi(p)$

(iii) For each $1 \geq p>p^{*}$ we have that $\psi(p)>\gamma b>\varphi(p)>\sigma(p)$.

Proof. In the Appendix. 
Figure 1 and Figure 2 depict two typical scenarios. Lemma (4) indicates that, for each $p>p^{*}$, if educated cohorts are able to invest in education, i.e., $f<\varphi(p)$ then also non-educated one can afford to invest in education since $\psi(p)>\varphi(p)$. Thus, the skill premium condition will be binding and consequently the long run equilibrium will be $p=p^{* *}$, where $p^{* *}$ solves $\sigma\left(p^{* *}\right)=f$. $p^{* *}$ is the value such that agents are indifferent between investing in education or not. Further, long run relative wealth converges towards unity. Simple algebra shows that $\frac{\partial p^{* *}}{\partial f}<0$ and $\frac{\partial p^{* *}}{\partial \eta}>0$.

Consider the case of $0<p \leq p^{*} \cdot \varphi(p)>f>\psi(p)$ is a steady state equilibrium. On the other hand neither $\varphi(p)<f$ nor $f<\psi(p)$ are steady state equilibria. In the former case skilled cohorts can no longer afford to invest in education. In the latter case unskilled cohorts can afford to invest in education. We can state the following proposition which summarises these results.

Proposition 5 In the steady state equilibrium one of the following conditions is satisfied: (i) $\varphi(p) \geq f \geq \psi(p)$

(ii) $p=p^{* *}$, where $\sigma\left(p^{* *}\right)=f$

\section{Proof. In the text.}

In case (i) of Proposition 5 the economy is characterised by persistent inequality. Notice that there is a continuum of equilibria satisfying this condition. Case (ii) describes a trickle down phenomenon where wealth inequality converges towards zero. We further investigate the possibility of a trickle down event restricting our attention to the case where $f<b \gamma$ and studying the properties of $\psi(p)$.

The locus of $\psi(p)$ predetermines the possibility of trickle down. The shape of $\psi(p)$ strongly depends on the parameters $\rho, b$ and $\eta$. The next lemma investigates some properties of $\psi(p)$.

Lemma 6 (i) if $1>\rho>0$, then $\lim _{p \rightarrow 0+} \psi(p)=\gamma b$; if $\rho=0$, then $\lim _{p \rightarrow 0+} \psi(p)=(1-\eta) \gamma b$; if $\rho<0$, then $\lim _{p \rightarrow 0+} \psi(p)=0$;

(ii) $\psi(p)$ is decreasing in $a=\frac{\eta}{1-\eta}$ and increasing in $b$; it is increasing in $\rho$ for $p \in\left(0, \frac{1}{2}\right)$ and decreasing in $\rho$ for $p \in\left(\frac{1}{2}, 1\right)$.

Proof. See the Appendix.

Lemma 6 states that, if $p<\frac{1}{2}$, then an increase in the factor substitutability increases the probability of a trickle down. Further, a larger bequest $b$ increases the probability of a trickle 


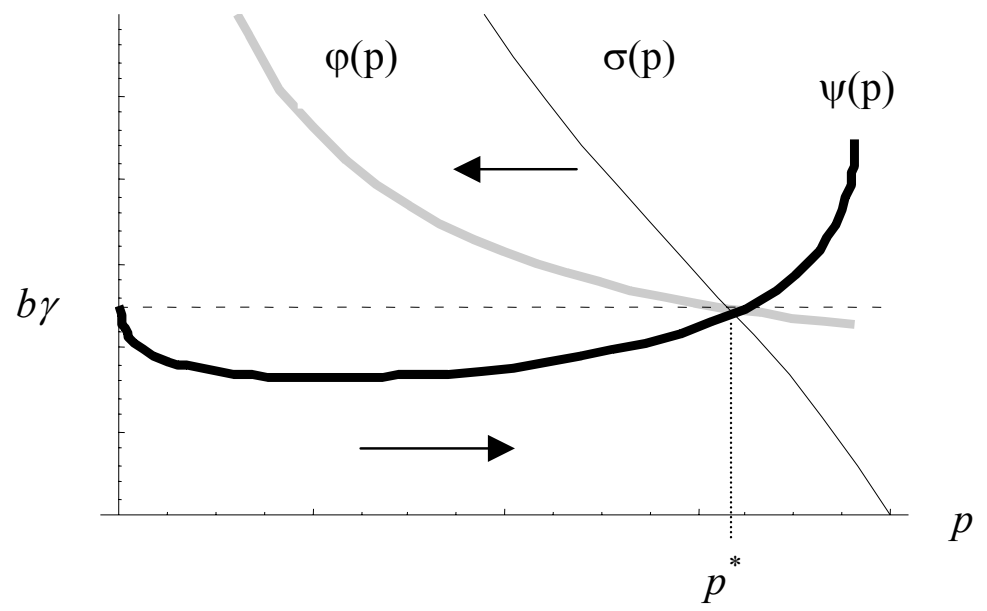

Figure 1: $\sigma(p), \varphi(p)$ and $\psi(p)$ as a function of $p$ where $\rho>0$.

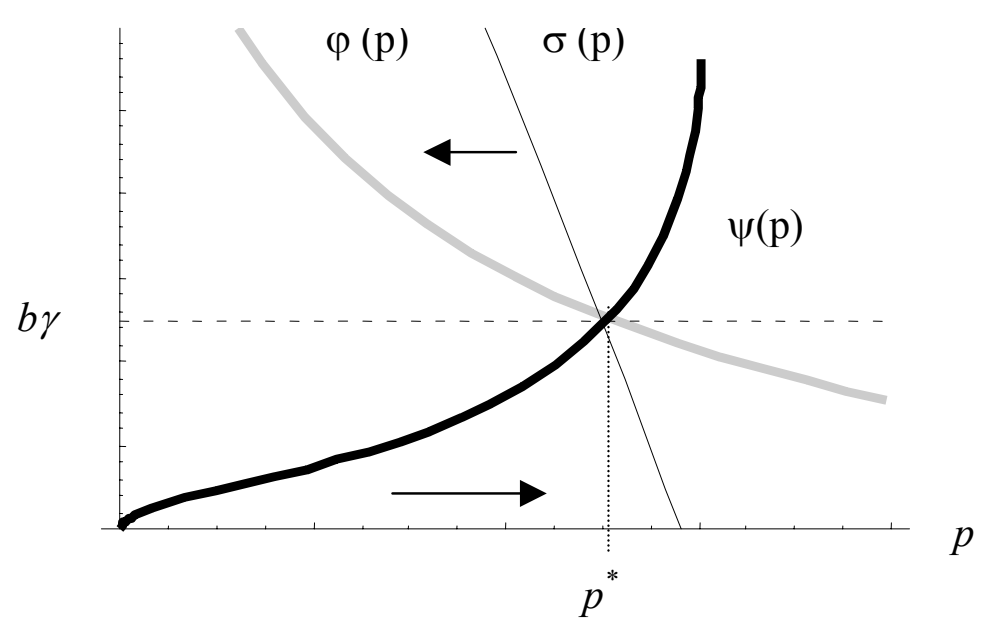

Figure 2: $\sigma(p), \varphi(p)$ and $\psi(p)$ where $\rho<0$. 
down, while an increase in $\eta$ decreases the probability of a trickle down.

The following proposition characterising the possibility of a trickle down can be proved.

Proposition 7 If skilled and unskilled workers are gross-substitutes, then there exists an open set of values for $f$ such that trickle down occurs irrespectively of the initial conditions. Further, this set is increasing in $\rho, b$ and decreasing in $\frac{\eta}{1-\eta}$. On the other hand, if skilled and unskilled workers are gross-complements, then there does not exist an open set of values of $f$ such that trickle down occurs irrespectively of the initial conditions.

Proof. Proposition 7 is a direct consequence of Lemma 6.

Proposition 7 states that if factors are gross-substitutes, then there exists always a sufficiently low value of the costs such that trickle down occurs irrespectively of the initial conditions. Further, we observe that the larger is $\rho$ and/or $b$ or the lower is $\eta$, the larger is the maximum value of $f$ which still yields a complete trickle down. On the other hand if factors are gross-complements then the possibility of a trickle down crucially depends on the initial conditions of the economy.

Further, Proposition 7 states that, the larger the relative importance of skilled workers in the aggregate production function, the lower the relative cost of education has to be such that a trickle down is feasible. Thus, technical progress, increasing the relative importance of skilled workers, makes a catching up of the poor more difficult, and consequently the persistence of inequality is increased. On the other hand, the larger the intergenerational transfer, the larger the relative cost of education can be such that a trickle down still occurs.

\subsection{Trickle down and aggregate wealth}

In this section we focus on the case where the relative cost of education is small enough, allowing for the trickle down equilibrium, i.e. $f \leq b \gamma$. A change in the relative size of educated individuals changes also the aggregate production level. In order to evaluate the aggregate effects of a trickle down in terms of production levels we write the steady state value of production as follows

$$
Y=N^{\frac{1}{\rho}}\left(\frac{b \gamma+1-\gamma-p f}{1+\beta}\right)^{\frac{\gamma}{1-\gamma}}
$$

The following result holds. 
Proposition 8 As long as $f \leq b \gamma$ and $\gamma \leq \frac{1}{2}$, if there are multiple long run equilibria, then there exists an negative relationship between aggregate production and inequality.

Proof. See the Appendix.

According to Proposition 8, as long as the relative weight of capital in the aggregate production function is not too large, a trickle down event increases aggregate production. Furthermore, Proposition 8 states that in the case of multipe equilibria, equilibria can be ordered according to their production level, where aggregate production is larger the lower is inequality. Thus, inequality is also an indicator of economic development.

We are also interested in how aggregate production changes if $f$ and/or $b$ change. A change in the size of the intergenerational transfer or in the relative cost of education changes not only the occurrence of a trickle down event as shown in Proposition 7, but it also changes the long run production level. We can state the following proposition.

Proposition 9 The larger the size of the intergenerational transfer, the more likely a trickle down event is, and the larger the long run production in the case of trickle down. As long as $f \leq b \gamma$ and $\gamma \leq \frac{1}{2}$, the lower the relative cost of education, the more likely a trickle down event is, and the larger the long run production in the case of trickle down.

Proof. See the Appendix.

According to Proposition 9 a larger intergenerational transfer and a lower relative cost of education not only increases the likelihood of a trickle down event, but it also increases the long run production level if the trickle down occurs.

\section{Conclusion}

In this paper we investigated the mechanics through which wealth may, in the long run, trickle down from the rich to the poor. For sufficiently large values of the relative cost of education the model predicts endogenous and permanent separation of the population between the rich (skilled) and the poor (unskilled). For lower values of this cost wealth eventually trickles down from the rich to the poor. In particular, we found that the larger is the size of the intergenerational transfer or the lower is the relative importance of the skilled labour in the production function, the larger 
is the set of initial conditions such that trickle down eventually occurs. Furthermore, the model predicts that if factors are gross substitutes, then there exists always values for the relative cost of education which allows trickle down irrespectively of the initial conditions. Thus, for sufficiently low values of the relative cost, a unique, invariant equilibrium exists where inequality vanishes asymptotically. For larger values of the relative cost, besides the trickle down equilibrium, a continuum of equilibria appear, where inequality persists forever. On the other hand, if factors are gross complements, then the model predicts that for each level of the relative cost of education, trickle down equilibrium always coexists with a continuum of equilibria characterised by persistent inequality. In other words, the equilibrium outcome crucially depends on the initial conditions of the system.

We found that, as long as the relative importance of labour in the aggregation production function is not too large, aggregate output increases in the event of a trickle down. This shows that the reduction of inequality does not occur at the expense of aggregate wealth. Furthermore, in the case of multiple equilibria, these can be ordered according to the aggregate wealth. In particular, an negative relationship between aggregate wealth and inequality can be established. We showed also that an increase in the size of the intergenerational transfer and/or a reduction in the relative cost of eduction not only makes a trickle down event more likely, but it also increases the long run level of production if a trickle down event occurs.

\section{Appendix}

Proof of Lemma 1. Solving the model for the steady state we obtain

$$
\begin{gathered}
\frac{Y}{K}=\frac{1+\beta}{b \gamma+1-\gamma-H f} \\
E^{L}=Y b \gamma(1-\eta) \frac{1-\gamma}{1-\gamma-H f} \frac{L^{\rho-1}}{N} \\
E^{H}=Y b \gamma \frac{\eta(1-\gamma) \frac{H^{\rho-1}}{N}-f}{1-\gamma-H f}
\end{gathered}
$$


Whence (18).

In order to show that the fixed point is globally stable we take the first derivative of (17) with respect to $\xi_{t-1}$ :

$$
\frac{\partial \xi_{t}\left(\xi_{t-1}\right)}{\partial \xi_{t-1}}=b \gamma N^{2} \frac{1-\gamma+b \gamma-f p}{\left[(1-\gamma)(1-\eta)(1-p)^{\rho-1}\left(p \xi_{t-1}+1-b\right)+b \gamma N\right]^{2}}
$$

From (21) it is easy to see that the map is concave. Furthermore, evaluating (21) at the fixed point (18) we obtain

$$
\frac{\partial \xi_{t}(\widetilde{\xi})}{\partial \xi_{t-1}}=\frac{b \gamma}{1-\gamma+b \gamma-f p}
$$

and consequently $1>\frac{\partial \xi_{t}(\tilde{\xi})}{\partial \xi_{t-1}}>0$.

Proof of Lemma 2. First observe that

$$
\sigma(p)=(1-\gamma) \frac{a-\left(\frac{1}{p}-1\right)^{\rho-1}}{a+\left(\frac{1}{p}-1\right)^{\rho}},
$$

where $a=\frac{\eta}{1-\eta}$.

The proof of (i) is straightforward, since $a$ is increasing with respect to $\eta$, while the right hand side of (22) is increasing with respect to $a$.

A straightforward calculation shows that the derivative of $\sigma(p)$ is

$$
\sigma^{\prime}(p)=(1-\gamma) \frac{-\left(a p^{\rho-1}-(1-p)^{\rho-1}\right)^{2}+(\rho-1) a p^{\rho-2}(1-p)^{\rho-2}}{\left(a+\left(\frac{1}{p}-1\right)^{\rho}\right)^{2}}
$$

Since the numerator of (23) is the sum of two non positive terms, (ii) follows.

(iii) is straightforward once we notice that $\left(\frac{1}{p}-1\right)^{\rho}$ is increasing for $0<p<\frac{1}{2}$, and decreasing for $\frac{1}{2}<p<1$.

Proof of Lemma 3. (a) Since $\bar{E}_{t}=E_{t}^{H}$, condition (15) reduces to the following $E_{t}^{H}>\frac{f}{\gamma b} E_{t}^{H}$. (b) In this case, individuals belonging to an educated dynasty can afford to invest in education, while those belonging to a non educated dynasty cannot afford to invest in education. Using 
condition (15) and observing that $\bar{E}_{t}=p E_{t}^{H}+(1-p) E_{t}^{L}$ this statement reduces to the following $E_{t}^{H}>\frac{f}{\gamma b}\left(p E_{t}^{H}+(1-p) E_{t}^{L}\right)$ and $E_{t}^{L}<\frac{f}{\gamma b}\left(p E_{t}^{H}+(1-p) E_{t}^{L}\right)$. Rearranging these inequalities we yield the expression stated in the Lemma. (c) All individuals are uneducated, and further they cannot afford to invest in education, i.e. $E_{t}^{L}<\frac{f}{\gamma b} E_{t}^{L}$.

Proof of Lemma 4 and Lemma 6. In this appendix we describe formally the properties of $\varphi(p)$ and $\psi(p)$. For notational convenience, let us define $a=\frac{\eta}{1-\eta}$.

The following properties can be proved :

(i) $\widetilde{\xi}>\xi^{*}$, for each $f<\hat{f}_{2}(p)$, and $f>\hat{f}_{1}(p)$, where $\widetilde{\xi}=\xi^{*}$ for $f=\hat{f}_{1}(p)$ and $f=\hat{f}_{2}(p)$,

(ii) $\widetilde{\xi}>\xi^{* *}$ for each $\bar{f}_{1}(p)>f>\bar{f}_{2}(p)$, where $\widetilde{\xi}=\xi^{* *}$ for $f=\bar{f}_{1}(p)=\bar{f}_{2}(p)$ where

$$
\begin{gathered}
\hat{f}_{1,2}(p)=\frac{\gamma b+1-\gamma \pm \sqrt{(\gamma b+1-\gamma)^{2}-4 \gamma b \frac{1-\gamma}{N} \eta p^{\rho}}}{2 p} \\
\bar{f}_{1,2}(p)=\frac{1-\gamma \pm \sqrt{(1-\gamma)^{2}-4 p \gamma b \frac{1-\gamma}{N}(1-\eta)(1-p)^{\rho-1}}}{2 p}
\end{gathered}
$$

(iii) $\hat{f}_{2}(p)$ is increasing in a and in $b \in\left(0, \frac{1-\gamma}{\gamma}\right)$

(iv) $\lim _{p \rightarrow 0+} \hat{f}_{2}(p)=+\infty$

(v) $\hat{f}_{2}(p)$ is decreasing in $\rho$ for $p \in\left(0, \frac{1}{2}\right)$ and it is increasing in $\rho$ for $p \in\left(\frac{1}{2}, 1\right)$

(vi) $\bar{f}_{2}(p)$ is decreasing in a and increasing in $b$

(vii)

$$
\lim _{p \rightarrow 0+} \bar{f}_{2}(p)=\left\{\begin{array}{c}
\gamma b \text { for } \rho>0 \\
\frac{\gamma b}{1+a} \text { for } \rho=0 \\
0 \text { for } \rho<0
\end{array}\right.
$$

(viii) $\bar{f}_{2}(p)$ is increasing in $\rho$ for $p \in\left(0, \frac{1}{2}\right)$ and it is decreasing in $\rho$ for $p \in\left(\frac{1}{2}, 1\right)$

Consider first part (i). $\widetilde{\xi} \geq \xi^{*}$ can be rewritten as

$$
f^{2} p-f(1-\gamma+\gamma b)+\eta p^{\rho-1} \frac{(1-\gamma) \gamma b}{N} \geq 0
$$


whose roots are given by (24).

Consider part (ii). $\widetilde{\xi} \geq \xi^{* *}$ can be rewritten as

$$
f^{2} p-f(1-\gamma)+(1-\eta)(1-p)^{\rho-1} \frac{(1-\gamma) \gamma b}{N} \leq 0
$$

Notice that $\hat{f}_{2}(p)$ is increasing in $a$ and in $b$, for $b \in\left(0, \frac{1-\gamma}{\gamma}\right)$, while $\bar{f}_{2}(p)$ is decreasing in $a$.For notational convenience we define $\varphi(p)=\hat{f}_{2}(p)$ and $\psi(p)=\bar{f}_{2}(p)$.

Furthermore, simple but tedius calculus show that the minimum of $\psi(p)$ in the case where factors are gross substitutes is increasing in $\rho$.

Proof of Proposition 8. Taking the first derivative of (20) with respect to $p$ yields

$$
\begin{gathered}
\frac{\partial Y}{\partial p}=N^{\frac{1}{\rho}-1}\left[\eta p^{\rho-1}-(1-\eta)(1-p)^{\rho-1}\right]\left(\frac{b \gamma+1-\gamma-p f}{1+\beta}\right)^{\frac{\gamma}{1-\gamma}}+ \\
N^{\frac{1}{\rho}} \frac{\gamma}{1-\gamma}\left(\frac{b \gamma+1-\gamma-p f}{1+\beta}\right)^{\frac{\gamma}{1-\gamma}-1}\left(-\frac{f}{1+\beta}\right)
\end{gathered}
$$

From the skill premium condition (19) we have that $\sigma(p) \geq f$ and thus

$$
\frac{\partial Y}{\partial p} \geq N^{\frac{1}{\rho}} \frac{f}{(1-\gamma)(1+\beta)}\left(\frac{b \gamma+1-\gamma-p f}{1+\beta}\right)^{\frac{\gamma}{1-\gamma}-1}[b \gamma+1-2 \gamma-p f]
$$

where the RHS is positive as long as $\gamma \leq \frac{1}{2}$ and $f \leq b \gamma$.

Proof of Proposition 9. First notice that the equilibrium value $p^{* *}$ is independent of $b$. Taking the first derivative of (20) with respect to $b$, taking into account that $p^{* *}$ does not change, yields the result.

Taking the first derivative of (20) with respect to $f$ yields

$$
\frac{\partial Y}{\partial f}<0 \text { if } f \frac{\partial p^{* *}}{\partial f}(b \gamma+1-\gamma-p f-\gamma)-\gamma p<0
$$

since $f<b \gamma$ in order to make trickle down feasible and $\frac{\partial p^{* *}}{\partial f}<0$, a sufficient condition for (26) to be true is that $f \leq b \gamma$ and that $\gamma \leq \frac{1}{2}$. 


\section{References}

ACEMoglu, D. (2002), Directed Technical Change, Review of Economics Studies, 69, 781-809.

AGHION, P. and BOLTON, P. (1997), A Theory of Trickle-Down Growth and Development, Review of Economic Studies, 64, 151-172.

ANDREONI, J. (1989), Giving with Impure Altruism: Applications to Charity and Ricardian Equivalence, Journal of Political Economy, 97, 1447-1458.

BANERJEE, A. and NEWMAN, A. (1993), Occupational Choice and the Process of Development, Journal of Political Economy, 101, 274-298.

BARRO, R. (1974), Are Government Bonds Net Wealth?, Journal of Political Economy, 82, 1095-1117.

GALOR, O. and ZEIRA, J. (1993), Income Distribution and Macroeconomics, Review of Economic Studies, 60 (1), 35-52.

MATSUYAMA, K. (2000), Endogenous Inequality, Review of Economic Studies, 67, 743759 .

MOOKHERJEE, D. and RAY, D. (2002b), Is Equality Stable?, American Economic Review, 92, 253-259.

MOOKHERJEE D. and RAY D. (2003), Persistent Inequality, Review of Economic Studies, 70, 369-393.

PIKETTY, T. (1997), The Dynamics of the Wealth Distribution and the Interest Rate With Credit Rationing, Review of Economic Studies, 64, 173-189. 